\title{
EFEITO DE 6-BENZILAMINOPURINA SOBRE A PROPAGAÇÃO in vitro DE Mimosa caesalpiniifolia Benth. (Fabaceae) ${ }^{1}$
}

\author{
Rafaela Maria de França Bezerra ${ }^{2}$, Magdi Ahmed Ibrahim Aloufa ${ }^{3}$,Fúlvio Aurélio de Morais Freire ${ }^{4}$ e \\ Daysilene Dantas dos Santos ${ }^{5}$
}

\begin{abstract}
RESUMO - A Mimosa caesalpiniifolia, popularmente conhecida como sabiá, é uma espécie nativa da Caatinga que, em razão de suas inúmeras potencialidades, tem enfrentado um processo de exaustiva exploração, tornando iminente a necessidade de se utilizar alternativas sustentáveis que permitam a sua reposição em ambiente natural e a conservação de seu genótipo. A micropropagação tem sido considerada técnica promissora nesse sentido, pois viabiliza a produção de mudas em larga escala e com elevada sanidade. Citocininas como a 6-benzilaminopurina (BAP) são importantes nesse processo, pois influenciam consideravelmente o crescimento e a morfogênese in vitro e permitem a formação de bancos de germoplasma in vitro. O objetivo deste trabalho foi verificar a influência de concentrações de BAP sobre a indução de brotações in vitro em M. caesalpiniifolia. Segmentos cotiledonares obtidos de plântulas germinadas in vitro foram inoculados em meio de cultura WPM suplementados com seis concentrações de BAP: 0,$0 ; 4,44 ; 8,88 ; 17,76 ; 26,64 ;$ e 35,52 $\mu \mathrm{mol} / \mathrm{L}$. O delineamento experimental utilizado foi o inteiramente casualizado com seis tratamentos, três repetições e cada repetição composta por 10 unidades experimentais. Aos 30 dias, foram avaliados o número de explantes responsivos, o número de brotos por explante e a presença de calos, oxidação e contaminação. Os dados foram avaliados estatisticamente através da Análise de Correspondência e mediante o ajuste de Equações de Regressão. Verificou-se que a concentração de $17,76 \mu \mathrm{mol} / \mathrm{L}$ apresentou-se mais responsiva em relação à taxa de multiplicação e ao número de brotações, sendo, portanto, a concentração mais indicada para a propagação in vitro de M. caesalpiniifolia.
\end{abstract}

Palavras-chave: Sabiá; Micropropagação; Citocinina.

\section{EFFECT OF 6-BENZYLAMINOPURINE ON in vitro PROPAGATION OF Mimosa caesapiniifolia Benth. (Fabaceae)}

\begin{abstract}
The Mimosa caesalpiniifolia, popularly known as sabiá, is a native species belonging to Caatinga forest that due to its countless potentialities has faced a process of exhaustive exploration that makes imminent the need to use sustainable alternatives to its replacement in natural environment and the conservation of its genotype. Accordingly, micropropagation has been considered as a promising technique, since it allows the production of seedlings in large scale and high. The cytokinins, such as 6-benzylaminopurine (BAP), are important in this process, since they influence considerably the growth and morphogenesis in vitro morphogenesis allowing the formation on in vitro germplasm banks. The aim of this study was to investigate the influence of different concentrations of BAP on in vitro budding induction of M. caesalpiniifolia. Cotyledon segments obtained from in vitro germinated seedlings were inoculated in WPM supplemented with six BAP concentrations: $0.0 ; 4.44 ; 8.88 ; 17.76 ; 26.64$ and $35.52 \mu \mathrm{mol} / \mathrm{L}$. The experimental design was randomized with 6 treatments, 3 replications and each replication consisting of 10 experimental units. After 30 days, the following parameters have been evaluated: number of responsive explants, number of sprouts per explant and presence of callus,
\end{abstract}

\footnotetext{
${ }^{1}$ Recebido em 02.10.2012 aceito para publicação em 04.07.2014.

${ }^{2}$ Programa de Pós-Graduação em Desenvolvimento e Meio Ambiente, Universidade Federal do Rio Grande do Norte, Brasil. E-mail: <rafaela_maria@hotmail.com>.

${ }^{3}$ Departamento de Botânica, Ecologia e Zoologia, UniversidadeFederal do Rio Grande do Norte, Brasil. E-mail: <magdialoufal@gmail.com>.

${ }^{4}$ Departamento de Botânica, Ecologia e Zoologia, Universidade Federal do Rio Grande do Norte, Brasil. E-mail:<fulvio@cb.ufrn.br>.

${ }^{5}$ Graduanda em Ciências Biológicas. Universidade Federal do Rio Grande do Norte, Brasil. E-mail: <dayse_leny10@hotmail.com>.
} 
oxidation and contamination. The data were statistically analyzed by Correspondence Analysis and through adjusting Regression Equations. It was found that the concentration of 17.76 umol/L was more responsive with regard to multiplication rate and sprouts number. In this case, is the most suitable concentration for the in vitro propagation of M. caesalpiniifolia.

Keywords: Sabiá; Micropropagation; Cytokinin.

\section{INTRODUÇÃO}

A Caatinga é o único bioma exclusivamente brasileiro e, apesar de estar localizado em área de clima semiárido, caracterizado por baixas e irregulares precipitações pluviométricas, apresenta grande variedade de paisagens, relativa riqueza biológica e endemismos (ARRUDA, 2001). Não obstante essas peculiaridades, pouca atenção tem sido dada à conservação da paisagem da Caatinga e a contribuição da sua biota à biodiversidade do Brasil tem sido subestimada (SILVA et al., 2004).

Dados do Ministério do Meio Ambiente (BRASIL, 2011) apontam que aproximadamente $80 \%$ da Caatinga já sofreu alguma influência antrópica e que somente entre os anos 2002 e 2008 foram perdidos mais de

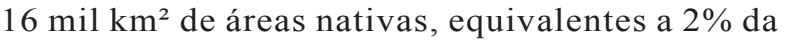
superfície total do bioma. Essa elevada pressão sobre os recursos florestais da Caatinga se deve, principalmente, à diversidade de potenciais que lhes são atribuídos, haja vista que várias espécies nativas desse bioma se destacam por sua aptidão forrageira, apícola, medicinal, madeireira e faunística (ARAÚJO FILHO et al., 2006).

Entre as espécies nativas da Caatinga que se destacam por seus múltiplos potenciais, encontra-se a Mimosa caesalpiniifolia Benth, popularmente conhecida como sabiá ou sansão-do-campo. Essa leguminosa de grande porte (5-8 $\mathrm{m}$ de altura) pertence à família Fabaceae e apresenta ampla distribuição ao longo dos Estados nordestinos, possuindo relevante valor econômico para a região (MOURA et al., 2006). A madeira do sabiá é vastamente utilizada na fabricação de postes, estacas e moirões, em razão de sua elevada resistência físico-mecânica (LORENZI, 2002). As suas folhas possuem alto valor forrageiro, e sua forragem é indicada para compor a dieta de manutenção de ruminantes, pois é nutritiva e palatável (SILVA, 2011). Apícola por excelência, o sabiá é apontado como um dos responsáveis por aumentar consideravelmente a produção de mel em algumas regiões do Nordeste brasileiro (MAIA-SILVA et al., 2012), e a infusão de sua casca tem sido prescrita para o tratamento de bronquites e ferimentos (CARVALHO, 2007).

Essa variedade de atributos têm exposto o sabiá a uma exploração frequente e, muitas vezes, exaustiva, o que torna evidente a necessidade de se utilizar alternativas sustentáveis que permitam tanto a sua reposição em ambiente natural quanto a conservação de seu genótipo. A micropropagação tem sido considerada alternativa promissora nesse sentido, pois viabiliza a produção de mudas em larga escala e com elevada qualidade fitossanitária em tempo e espaço reduzidos (GIRI et al., 2004; XAVIER, 2007). Além disso, essa técnica permite a formação de bancos de germoplasma in vitro, onde recursos genéticos de plantas podem ser armazenados por um período considerável sem a perda de sua viabilidade (SOUZA et al., 2009).

Através da micropropagação, fragmentos de tecidos vegetais vivos, denominados explantes, são retirados de plantas de interesse e cultivados em meio nutritivo definido, em condições assépticas (SERAFINI et al., 2001). A composição do meio de cultura é um dos fatores que mais influenciam a multiplicação e o desenvolvimento in vitro dos explantes, haja vista que o crescimento e morfogênese in vitro são fatores regulados pela interação e pelo balanço dos reguladores de crescimento no meio nutritivo (VILLA et al., 2007).

As citocininas são reguladores de crescimento que exercem importante papel na micropropagação, pois influenciam diretamente a expansão foliar, a quebra da dominância apical e a formação de gemas adventícias (POZO et al., 2005). Os tipos de citocininas e suas concentrações são os fatores que mais influenciam o sucesso da multiplicação in vitro, pois a necessidade dessas substâncias varia de acordo com a espécie a ser trabalhada e de seus níveis hormonais endógenos (CORDEIRO et al., 2004).

Entre as citocininas, a 6-benzilaminopurina (BAP) tem sido a mais indicada para promover a proliferação de partes aéreas e indução de gemas adventícias in

Revista Árvore, Viçosa-MG, v.38, n.5, p.771-778, 2014 
vitro (GRATTAPAGLIA; MACHADO, 1998). Diversos trabalhos vêm sendo desenvolvidos com o objetivo de analisar a influência de BAP sobre a propagação in vitro de espécies lenhosas nativas da caatinga, como Amburana cearensis (CAMPOS, 2013), Amburana acreana (FERMINO JUNIOR; PEREIRA, 2012), Erythrina velutina (COSTA et al., 2010) e Bauhinia cheilantha (GUTIERREZ et al., 2011), em que a utilização de concentrações adequadas dessa citocinina potencializou a multiplicação in vitro dessas espécies. Assim, o objetivo deste trabalho foi avaliar o efeito de diferentes concentrações da citocinina 6-benzilaminopurina (BAP) sobre a multiplicação in vitro de segmentos cotiledonares de M. caesalpiniifolia.

\section{MATERIAL E MÉTODOS}

Os ensaios foram realizados no Laboratório de Biotecnologia de Conservação de Espécies Nativas (LABCEN), da Universidade Federal do Rio Grande do Norte. Foram utilizados como explantes segmentos cotiledonares de plântulas germinadas in vitro com idade fisiológica de 30 dias. Para a indução da germinação in vitro, as sementes de $M$. caesalpiniifolia passaram pelo procedimento de quebra de dormência, que consistiu em escarificação mecânica com lixa número 100 até a remoção de aproximadamente $60 \%$ de seu tegumento, seguida por imersão em água destilada à temperatura ambiente por $12 \mathrm{~h}$, conforme recomendação de Zaidan e Barbedo (2004).

Posteriormente, as sementes passaram pelo processo de desinfestação em capela de fluxo laminar, em que foram imersas em álcool 70\% (v/v) por $1 \mathrm{~min}$, em solução de hipoclorito de sódio $1 \%$ por 10 min e em seguida submetidas a três enxagues em água destilada e autoclavada, com duração de 10 min cada. Em seguida, as sementes foram inoculadas em frascos contendo $60 \mathrm{~g}$ de vermiculita expandida e umedecida com $30 \mathrm{~mL}$ de água destilada, mantidas em sala de crescimento com temperatura de $24^{\circ} \mathrm{C}$, fotoperíodo de $6 \mathrm{~h}$ e intensidade luminosa de $45 \mu \mathrm{mol} . \mathrm{m}^{-2} . \mathrm{s}^{-1}$.

Trinta dias após a inoculação, as plântulas foram submetidas a um processo de lavagem, para que resquícios do substrato utilizado para a germinação fossem completamente eliminados. Para isso, foram imersas em álcool 70\% durante $1 \mathrm{~min}$, em hipoclorito de sódio $1 \%$ durante $5 \mathrm{~min}$, e enxaguadas em água destilada e autoclavada em três banhos, com duração de 10 min cada. Após esse procedimento, segmentos nodais medindo aproximadamente $2 \mathrm{~cm}$ foram inoculados em frascos contendo $25 \mathrm{~mL}$ de meio de cultura WPM (LLOYD; MCCOWN, 1980), com pH ajustado para 5,8 e suplementado com $30 \mathrm{gL}^{-1}$ de sacarose, $7 \mathrm{gL}^{-1}$ de ágar da marca comercial Vetec e seis diferentes concentrações de BAP: 0,$0 ; 4,44 ; 8,88 ; 17,76 ; 26,64$; e $35,52 \mu \mathrm{mol} / \mathrm{L}$.

O delineamento experimental utilizado foi o inteiramente casualizado com seis tratamentos e três repetições, sendo cada repetição composta por 10 unidades experimentais (frascos). Os frascos inoculados foram mantidos em sala de crescimento com as condições ambientais semelhantes às descritas anteriormente. Após 30 dias da inoculação, procedeu-se à avaliação, quando foram observadas as seguintes variáveis: número de explantes responsivos, isto é, que formaram brotos e número de brotações por explante, além do total de explantes que apresentaram calos, oxidação e contaminação.

Os resultados das variáveis explantes brotados, explantes com calos, explantes oxidados e explantes contaminados foram submetidos à Análise de Correspondência utilizando o software estatístico PAST versão 2.6 (HAMMER et al., 2001). Os dados dos números de brotações por explante foram transformados em $(x+0,5)^{0,5}$ e ajustados com os dados de concentrações de BAP em um modelo não linear, utilizando-se o pacote nls do programa estatístico $\mathrm{R}$ (2008). O pacote nls calcula os erros-padrão dos parâmetros estimados com base na teoria de verossimilhança, sob a suposição de erros normalmente distribuídos. O modelo foi ajustado em um polinômio de segundo grau, em que a variável $\mathrm{x}$ foram as concentrações de BAP, enquanto $\mathrm{Y}$, os números de brotações por explante.

\section{RESULTADOS}

As diferentes concentrações de BAP influenciaram significativamente todas as variáveis analisadas. A análise de correspondência envolvendo as variáveis presença de brotações, presença de calos, presença de oxidação e presença de contaminação pode ser visualizada no diagrama de ordenação apresentado na Figura 1. Os autovalores dos dois eixos de ordenação foram: 0,$0857 ; 0,0176$; e 0,0123, sendo responsáveis, respectivamente, por $74,08 \%, 15,22 \%$ e $10,68 \%$ da variância total de seis concentrações de BAP, com relação às variáveis analisadas.

Revista Árvore, Viçosa-MG, v.38, n.5, p.771-778, 2014 


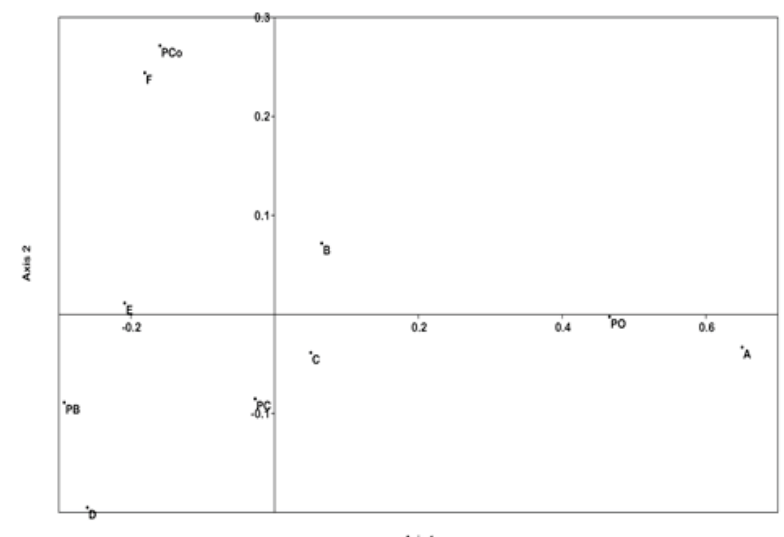

Figura 1 - Diagrama de ordenação dos eixos da Análise de Correspondência das variáveis Presença de Brotação (PB), Presença de Oxidação (PO), Presença de Calos (PC) e Presença de Contaminação (PCo) nas distintas concentrações de $\mathrm{BAP}(\mathrm{A}=0,00 \mu \mathrm{mol} / \mathrm{L} ; \mathrm{B}=4,44$ $\mu \mathrm{mol} / \mathrm{L} ; \mathrm{C}=8,88 \mu \mathrm{mol} / \mathrm{L} ; \mathrm{D}=17,76 \mu \mathrm{mol} / \mathrm{L} ; \mathrm{E}$ $=26,64 \mu \mathrm{mol} / \mathrm{L} ;$ e $\mathrm{E}=35,52 \mu \mathrm{mol} / \mathrm{L}$ ).

Figure 1 - Ordination diagram of the axes of Correspondence Analysis for variables: Presence of Sprouting (PS), Presence of Oxidation (PO), Presence of Calluses $(P C)$ and Presence of Contamination (PCo) for the different BAP concentrations $(A=0.00 \mu \mathrm{mol} / \mathrm{L}$; $B=4.44 \mu \mathrm{mol} / \mathrm{L} ; C=8.88 \mu \mathrm{mol} / \mathrm{L} ; D=17.76 \mu \mathrm{mol} /$ $L ; E=26.64 \mu \mathrm{mol} / \mathrm{L}$ and $E=35.52 \mu \mathrm{mol} / \mathrm{L})$.

A Figura 1 mostra que as variáveis Presença de Brotações e Presença de Oxidação apresentaram maior correlação com o primeiro eixo de ordenação (Axis 1), enquanto as variáveis Presença de Calos e Presença de Contaminação exibiram maior correlação com o segundo eixo (Axis 2).

No primeiro eixo de ordenação, a variável Presença de Brotações apresentou maior correlação com a concentração de $17,76 \mu \mathrm{mol} / \mathrm{L}$ de BAP, em que atingiu o ápice em número de explantes responsivos para a formação de brotos. Já a variável Presença de Oxidação apresentou correlação com o decréscimo de BAP, mostrando-se mais correlacionada a ausência da citocinina no meio nutritivo.

No segundo eixo de ordenação, percebeu-se que a variável Presença de Calos apresentou maior correlação com a concentração de $8,88 \mu \mathrm{mol} / \mathrm{L}$ de BAP. Em contrapartida, a menor correlação ocorreu com as menores dosagens desse regulador de crescimento $(0,0 ;$ e 4,44; $\mu \mathrm{mol} / \mathrm{L})$. A variável Presença de Contaminação teve relação direta com a concentração de BAP, ou seja, houve maior correlação dessa variável com a maior concentração do regulador de crescimento $(35,52 \mu \mathrm{mol} / \mathrm{L})$, e essa taxa apresentou decréscimo à medida que diminuiu a concentração de BAP.

Em relação ao número de brotações, os dados do modelo, ajustados através da função polinomial de segundo grau, indicaram alto grau de relação, com um coeficiente de determinação $\left(\mathrm{R}^{2}\right)$ de 0,85 . Os resultados dos parâmetros do modelo, com os respectivos intervalos de confiança e significância estatística, encontram-se na Tabela 1. A curva de resposta (Figura 2) apresenta-se ascendente até a concentração de 17,76 $\mu \mathrm{mol} / \mathrm{L}$ de BAP, em que atinge o ápice de brotações, seguido pelo declínio quando os explantes são submetidos a concentrações superiores à supracitada.

\section{DISCUSSÃO}

A organogênese in vitro de brotos ocorreu em todas as concentrações utilizadas do regulador de crescimento BAP, inclusive na sua ausência, o que sugere a existência de concentrações endógenas de citocinina suficientes para induzir o brotamento dos segmentos cotiledonares. A adição de BAP ao meio de cultura em concentrações de até $17,76 \mu \mathrm{mol} / \mathrm{L}$ ampliou tanto o número de explantes responsivos quanto o número de brotações por explante, seguido pelo decréscimo em ambas as variáveis, mediante o incremento na concentração dessa citocinina.

Tabela 1 - Estimativas dos parâmetros da equação polinomial ajustada para exprimir a relação entre as concentrações de BAP e o número de brotações em M. caesalpiniifolia Benth.

Table 1 - Estimates of the polynomial equation parameters adjusted to express the relation between BAP concentrations and number of sprouts in M. caesalpiniifolia Benth.

\begin{tabular}{cccccc}
\hline & $\begin{array}{c}\text { Valor do } \\
\text { parâmetro }\end{array}$ & $\begin{array}{c}\text { Erro- } \\
\text { padrão }\end{array}$ & \multicolumn{2}{c}{$\begin{array}{c}\text { Valor } \\
\text { de t }\end{array}$} & \multicolumn{2}{c}{$\begin{array}{c}\text { Valor } \\
\text { probabilístico }\end{array}$} & \multicolumn{2}{c}{$\begin{array}{c}2,5 \% \\
9,5 \%\end{array}$} \\
\hline A & $-0,005$ & 0,001 & $-3,853$ & 0,031 & $-0,009$ \\
B & 0,188 & 0,045 & 4,201 & 0,025 & 0,046 \\
CONSTANTE & 1,558 & 0,301 & 5,185 & 0,014 & 0,332 \\
\hline
\end{tabular}

Revista Árvore, Viçosa-MG, v.38, n.5, p.771-778, 2014 


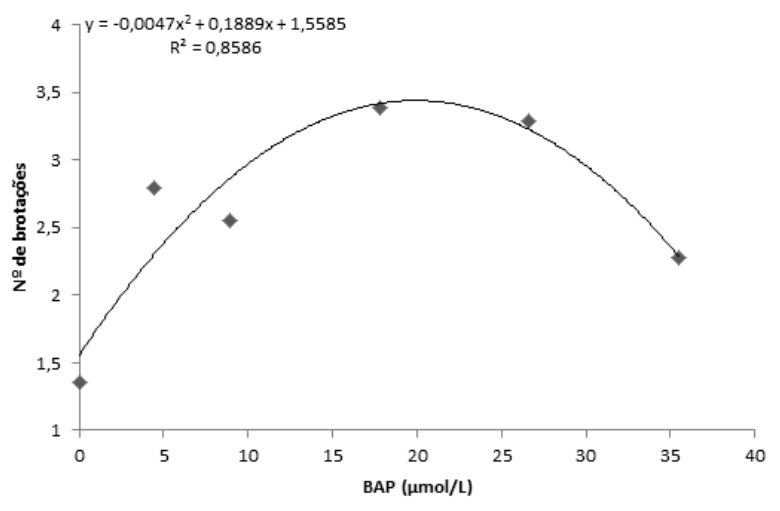

Figura 2 - Efeito fisiológico de concentrações de BAP $(\mu \mathrm{mol} / \mathrm{L})$ no número médio de brotações de M. caesalpiniifolia Benth.

Figure 2 - Physiological effect of BAP concentrations $(\mu \mathrm{mol} / \mathrm{L})$ in the average number of sprouts of M. caesalpiniifolia Benth.

Efeitos semelhantes foram observados por Sato et al. (2004) e Schottz et al. (2007), que verificaram aumento na taxa de multiplicação em Swietenia macrophylla e Gallesia gorazema diretamente relacionado ao incremento da concentração de BAP até determinada concentração considerada ótima, seguida por decréscimo nessa taxa ao submeter os explantes a concentrações superiores dessa citocinina.

Ainda corroborando os dados desta pesquisa, Kielse et al. (2009) e Costa et al. (2010) observaram a formação de brotos em Parapiptadenia rigida, espécie florestal também pertencente à família Fabaceae; e em Erythrina velutina, mesmo em meio de cultura sem a presença de reguladores de crescimento. No entanto, os segmentos cotiledonares inoculados em meio de cultura contendo BAP apresentaram maiores percentuais de brotações.

Em relação ao número de brotações por explante, a relação do efeito de concentrações de BAP sobre essa variável encontrada nesta pesquisa concorda com resultados de Fermino Júnior e Pereira (2012), ao avaliarem o número de brotos de Amburana acreana sob a influência de diferentes concentrações desse regulador de crescimento. Esses autores verificaram que o número de brotações apresentou-se ascendente com o aumento na concentração de BAP até atingir o ponto máximo em $17,76 \mu \mathrm{mol} / \mathrm{L}$, com consecutivo decréscimo à medida que doses dessa citocinina foram aumentadas.
Resultados semelhantes foram obtidos por Gutiérrez (2011), que demonstrou relação quadrática entre essas duas variáveis em explantes de Bauhinia cheilantha, espécie leguminosa nativa da Caatinga. $\mathrm{Na}$ referida pesquisa, o número de brotações em segmentos cotiledonares apresentou-se ascendente até atingir valor máximo na concentração de BAP, e a partir dessa concentração houve tendência à diminuição na formação de brotos para o explante segmento cotiledonar.

Cordeiro et al. (2004) atribuíram esse efeito benéfico do BAP na multiplicação de brotações à influência desse regulador de crescimento na divisão celular e na liberação das gemas auxiliares inibidas pela dominância apical. No entanto, Grattapaglia e Machado (1998) explicaram que essa tendência de diminuição na taxa de multiplicação e no número de brotações por explante com a adição de concentrações superiores de BAP pode estar ligada ao fato de que as citocininas estimulam a maior produção de partes aéreas até determinada concentração, o que varia de acordo com a espécie e, a partir desta, ocorre efeito tóxico, caracterizado pela falta de alongamento das culturas.

No que diz respeito à oxidação dos explantes, os resultados sugeriram que existe razão inversamente proporcional entre a variável e os níveis de tratamentos, ou seja, quanto menor a concentração de BAP adicionado ao meio de cultura, maior a presença de oxidação. Esses resultados foram corroborados por Bassan et al. (2006), ao verificarem que na ausência de reguladores de crescimento os explantes de Peltophorum dubium apresentaram os maiores índices de oxidação (entre 83 e 94\%). Apesar de o meio sem regulador de crescimento apresentar tendência à maior taxa de oxidação, a adição de BAP ao meio de cultura não teve efeito significativo na presença de oxidação em pesquisa realizada por Sato et al. (2004), ao estabelecerem protocolos para controle de oxidação de Gallesia gorazema.

Resultados semelhantes foram obtidos ainda por Soares et al. (2011), ao observarem a presença de oxidação em explantes de Ochroma pyramidale inoculados em meio de cultura sem a adição de reguladores de crescimento. Bassan et al. (2006) explicaram que a ocorrência de compostos fenólicos pode estar ligada a processos de regulação de crescimento, os quais, dependendo da concentração endógena no tecido, induzem à síntese dos compostos polifenólicos. 
A oxidação ocorre em função da liberação de compostos fenólicos in vitro, geralmente devido a danos causados nas células durante a excisão dos explantes. Esses compostos fenólicos são oxidados pelas enzimas polifenases, produzindo substâncias tóxicas, modificando a absorção de metabólitos e inibindo o crescimento dos explantes (ANDRADE et al., 2000), o que justifica a menor frequência de brotações em explantes com elevados índices de oxidação.

A variável Formação de Calos apresentou menor correlação com a ausência de BAP, e essa correlação maximiza-se à medida que eleva a concentração de citocinina até a concentração de $8,88 \mu \mathrm{mol} / \mathrm{L}$, em que atinge a taxa máxima de calogênese. A adição de concentrações superiores de BAP proporcionou decréscimo na formação de calos com posterior estabilização entre 17,76 e $26,64 \mu \mathrm{mol} / \mathrm{L}$ de BAP.

Segundo Guerra e Nodari (2006), a formação de calos ocorre quando há equilíbrio entre as concentrações de auxina e citocinina. Desse modo, pode-se afirmar que a concentração de $8,88 \mu \mathrm{mol} / \mathrm{L}$ de BAP foi a que proporcionou maior equilíbrio entre esses dois reguladores de crescimento em explantes de sabiá. Incrementos nessa concentração repercutiram na diminuição da calogênese, no entanto proporcionaram maior taxa de brotações dos explantes, o que pode ser justificado pelo aumento na relação citocinina/auxina.

Tendências semelhantes foram observadas em ensaios de micropropagação de Schizolobium amazonicum (CORDEIRO et al., 2004) e P. rigida (KIELSE et al., 2008). A formação de calos ocorreu com menor frequência no meio nutritivo sem reguladores de crescimento, e o acréscimo de citocinina em concentrações ascendentes proporcionou tendência de crescimento na taxa de formação de calos. Grattapaglia e Machado (1998) alertaram para o fato de que, apesar de a formação de calos na base do explante ser fenômeno bastante comum em espécies lenhosas, esse processo é considerado indesejável na micropropagação, pois podem comprometer a rizogênese e o crescimento da parte aérea.

No que concerne às taxas de contaminação, embora ainda seja deficiente o número de pesquisas que investiguem a influência do BAP sobre a multiplicação de agentes contaminantes in vitro, autores como Costa et al. (2007) e Rosa (2009) também verificaram tendência a maiores taxas de contaminação com o aumento na concentração desse regulador de crescimento em explantes de Lippia Sidoides e Mimosa scabrella, respectivamente. Nesse sentido, Rosa (Op. cit.) sugere que a citocinina BAP a partir de certas concentrações, pode contribuir significativamente na multiplicação de microrganismos.

Tanto a contaminação da superfície dos tecidos de folhas, gemas e segmentos nodais quanto a endógena (presente no interior dos tecidos) constituem desafios a serem superados na micropropagação de plantas, que visa a obtenção de mudas livres de agentes contaminantes. A contaminação constitui um dos maiores problemas enfrentados na fase inicial de estabelecimento do explante in vitro (TEIXEIRA, 2001), por isso é fundamental que se realize uma etapa de assepsia eficiente previamente à inoculação in vitro.

\section{CONCLUSÃO}

A concentração ideal de BAP para o incremento na taxa de multiplicação, bem como para o número de brotações em M. caesalpiniifolia, é de 17,76 $\mu \mathrm{mol} /$ L. Concentrações superiores desse regulador de crescimento reduzem significativamente o número de brotações em segmentos cotiledonares dessa espécie.

\section{AGRADECIMENTOS}

Ao Deutscher Akademischer Austauschdienst (DAAD), pela bolsa de pesquisa concedida à primeira autora; e ao Laboratório de Biotecnologia de Conservação de Espécies Nativas (LABCEN/UFRN), pelos suportes físico e estrutural necessários ao desenvolvimento desta pesquisa.

\section{REFERÊNCIAS}

ANDRADE, M. W. et al. Micropropagação da aroeira (Myracrodruon urundeuva Fr. All).

Ciência e Agrotecnologia, v.24, n.1, p.174$180,2000$.

ARAÚJO FILHO, J. A. et al. Sistema agrossilvipastoril - Embrapa Caprinos. In: LIMA, G. F. C. et al. Criação familiar de caprinos e ovinos no Rio Grande do Norte: orientações para viabilização do negócio rural. Natal: EMATER-RN, EMPARN, Embrapa Caprinos, 2006. p.193-210.

ARRUDA, M. B. Ecossistemas brasileiros. Brasília: IBAMA, 2001. 
BASSAN, J. S. et al. Oxidação fenólica, tipo de explante e meios de cultura no estabelecimento in vitro de canafístula (Peltophorum dubium).

Ciência Florestal, v.16, n.4, p.381-390, 2006.

BRASIL. Ministério do Meio Ambiente.

Monitoramento do desmatamento dos biomas brasileiros por satélite: monitoramento do bioma caatinga. Brasília, DF: Centro de Sensoriamento Remoto, 2011. Relatório Técnico. Disponível em: < http://www.mma.gov.br/ estruturas/sbf_chm_rbbio/_arquivos/ relatorio tecnico caatinga 20082009 72.pdf>. Acesso em: 25 jun. 2012.

CAMPOS, V. C. A. et al. Micropropagação de umburana de cheiro. Ciência Rural, v.43, n.4, p.639-644, 2013.

CARVAlHo, P. E. Sabiá - Mimosa caesalpiniifolia. Colombo: 2007. p.1-9. (Circular Técnica, 135)

CORDEIRO, I. M. C. C. et al. Efeito de BAP sobre a proliferação de brotos in vitro de Schizolobium amazonicum Huber ex Ducke (paricá). Cerne, v.10, n.1, p.118-124, 2004.

COSTA, A. S. et al. Estabelecimento de alecrimpimenta in vitro. Horticultura Brasileira, v. 25, n. 1, p.68-72, 2007.

COSTA, G. M.; NEPOMUCENO, C. F.; SANTANA, J. R. F. Propagação in vitro de Erythrina velutina. Ciência Rural, v.40, n.5, p.10901096, 2010.

FERMINO JUNIOR, P. C. P.; PEREIRA, J. E. S. Germinação e propagação in vitro de Cerejeira (Amburana acreana (ducke) a.c. Smith - Fabaceae). Ciência Florestal, v.22, n.1, p.1-9, 2012.

GIRI, C. C.; SHYAMKUMAR, B.; ANJANEYULU, C. Progress in tissue culture, genetic 283 transformation and applications of biotechnology to trees: an overview. Trees, v.18, p.115-135, 2004.

GRATTAPAGLIA, D.; MACHADO, M. A.

Micropropagação. In: TORRES, A. C.; CALDAS, L. S.; BUSO, J. A. (Ed.). Cultura de tecidos e transformação genética de plantas. Brasília: Embrapa-SPI $\backslash$ Embrapa CNPH, 1998. v.1. p.183-260.
GUERRA, M. P.; NODARI, R. O. Apostila de Biotecnologia 1: cultura de tecidos vegetais. Florianópolis: UFSC/Steinmacher, 2006. 41p.

GUTIÉRREZ, M. et al. Regeneração in vitro via organogênese direta de Bauhinia cheilantha. Ciência Rural, v.41, n.2, p.260-265, 2011.

HAMMER, Ø.; HARPER, D. A. T.; RYAN, P. D. PAST: Paleontological statistics software package for education and data analysis. Paleontologia Electronica, v.1, p.1-9, 2001.

KIELSE, P. et al. Regeneração in vitro de Parapiptadenia rigida. Ciência Rural, v.39, n.4, p.1098-1104, 2009.

LLOYD, G.; McCOWN, B. Commercially-feasible micropropagation of mountain laurel, Kalmia latifolia, by use of shoot-tip culture.

Combined Proceedings of International Plant Propagators Society, v.30, p.421-427, 1980 .

LORENZI, H. Árvores brasileiras: manual de identificação e cultivo de plantas arbóreas nativas do Brasil. 2. ed. Nova Odessa: Instituto Plantarum, 2002. v.2. 382p.

MAIA-SILVA, C. et al. Guia de plantas visitadas por abelhas na caatinga. Fortaleza: Fundação Brasil Cidadão, 2012.

MOURA, O. N. et al. Distribuição da biomassa e nutrientes na área de Mimosa caesalpiniifolia Benth. Revista Árvore, v.30, n.6, p.877-884, 2006.

POZO, J. C. D. et al. Hormonal control of the plant cell cycle. Biologia Plantarum, v. 123, p. 173-183, 2005.

R. DEVELOPMENT CORE TEAM. R: A language and environment for statistical computing. Vienna: R Foundation for Statistical Computing. 2008. Disponível em: http://www.R-project.org/.

ROSA, F. C. Superação de dormência de sementes e cultivo in vitro de Bracatinga (Mimosa scabrella Benth.). 2009. 52f. Dissertação (Mestrado em Engenharia Florestal)-Universidade Federal de Santa Maria, Santa Maria, 2009.

Revista Árvore, Viçosa-MG, v.38, n.5, p.771-778, 2014 
SERAFINI, L. A.; BARROS, N. M.; AZEVEDO, J. L. Biotecnologia: princípios e aplicações. In: SERAFINI, L. A.; BARROS, N. M.; AZEVEDO, J. L. (Ed.). Biotecnologia na agricultura e na agroindústria. Guaíba: Agropecuária, 2001. p.25-74.

SATO, A. Y. et al. Controle de contaminação e oxidação na micropropagação do Pau D'alho (Gallesia gorazema MOQ.). Agropecuária Técnica, v. 25, n. 2, p. 65-70, 2004.

SCHOTTZ, E. S. et al. In vitro multiplication of Swietenia macrophylla King (Meliaceae) from juvenile shoots. Ciência Florestal, v.17, n.1, p.109-117, 2007.

SILVA, J. M. C. et al. (Org.). Biodiversidade da caatinga: áreas e ações prioritárias para a conservação. Brasília: Ministério do Meio Ambiente: Universidade Federal de Pernambuco, 2004. 382p.

\section{SILVA, M. A. Caracterização de} leguminosas arbustivo-arbóreas em Pernambuco. 2011. 129f. Tese (Doutorado em Zootecnia) - Universidade Federal Rural de Pernambuco, Recife, 2011.

SOARES, F. P. Taxa de multiplicação e efeito residual de diferentes fontes de citocinina no cultivo in vitro de Hancornia speciosa Gomes. Ciência e Agrotecnologia, v.35, n.1, p.152-157, 2011.

SOUZA, A. S. et al. Preservação de Germoplasma Vegetal, com ênfase na conservação in 67 vitro de variedades de mandioca. Cruz das Almas: Embrapa Mandioca e Fruticultura Tropical, 2009.p.11-24. (Circular Técnica)

TEIXEIRA, J. B. Limitações ao processo de cultivo in vitro de espécies lenhosas. Brasília: Embrapa-Recursos Genéticos e Biotecnologia, 2001.

VILLA, F. et al. Influência do carvão ativado e BAP na multiplicação in vitro de duas frutíferas de clima temperado. Revista Ceres, v.54, n.312, p.118-124, 2007.

XAVIER, A.; OTONI, W.C.; PENCHEL, R.M. Micropropagação e enxertia in vitro de espécies florestais. In: BORÉM, A. (Ed). Biotecnologia Florestal. Viçosa, MG: Universidade Federal de Viçosa, 2007. p55-74.

ZAIDAN, L. B. P.; BARBEDO, C. J. Quebra de dormência em sementes. In: FERREIRA, A.G; BORGHETTI, F. Germinação: do básico ao aplicado. Porto Alegre: Artmed, 2004. p. 135-148. 